\title{
A Quantitative Analysis of Patients' Narratives of Heart Failure
}

\author{
Sabita Acharya, Barbara Di Eugenio, Andrew Boyd, Richard Cameron, \\ Karen Dunn Lopez, Pamela Martyn-Nemeth, Debaleena Chattopadhyay, \\ Pantea Habibi, Carolyn Dickens, Haleh Vatani, Amer Ardati \\ University of Illinois at Chicago \\ Chicago, Illinois \\ sachar4, bdieugen, boyda, rcameron, kdunn12, pmartyn, \\ debchatt, phabib4, cdickens, hvatan2, aardati@uic.edu
}

\begin{abstract}
Patients with chronic conditions like heart failure are most likely to be re-hospitalized. One step towards avoiding re-hospitalization is to devise strategies for motivating patients to take care of their own health. In this paper, we perform a quantitative analysis of patients' narratives of their experience with heart failure and explore the different topics that patients talk about. We compare two different groups of patients- those unable to take charge of their illness, and those who make efforts to improve their health. We will use the findings from our analysis to refine and personalize the summaries of hospitalizations that our system automatically generates.
\end{abstract}

\section{Introduction}

Patients with heart failure are responsible for around $95 \%$ of their chronic illness care and their daily decisions have a huge impact on their quality of life (Funnell, 2000). Studies have shown that the patients' perspective is essential for patient education (Shapiro, 1993) and that engaging the patients in their own care reduces hospitalizations and prevents further deterioration of their health (Riegel et al., 2011; McGinnis et al., 2013).

We are engaged in a large, long-term project that aims to improve patient discharge instructions with a personalized and comprehensible summary of their hospital stay that is informed by the perspectives of the three main stake-holders: doctors, nurses, and patients. Over the last few years, we have developed and implemented a framework for summarizing heterogeneous information (textual discharge notes from the doctor, structured information from the nurses) and providing explanations for difficult medical terms (Di Eugenio et al., 2014; Acharya et al., 2016, 2018, 2019). Figure 1 shows a part of a summary that is generated by our system.

You were admitted for acute subcortical
cerebrovascular accident. During your hospitaliza-
tion, you were monitored for chances of ineffec-
tive cerebral tissue perfusion, risk for falls, problem
in verbal communication and walking. We treated
difficulty walking related to nervous system disorder with
body mechanics promotion. [...] As a result, fall prevention
behavior and [...] improved slightly. With your nurse and
doctors, you learned about disease process and medication.
Follow-up: Can follow-up with General Neurology clinic
and Medicine clinic as outpatient if desired.

Figure 1: Portion of a summary generated by our system. Underlined terms provide a lay language definition when clicked.

A high level flowchart of the algorithm is shown in Appendix A. At this point, most inputs in pink are available for and used by the algorithm other than strengths and concerns of the patient (uncovering which is part of the focus of this paper). Specifically, the current version of our system uses the following personalization features:

A) Participation in self-care: The Patient Activation Measure (PAM) (Hibbard et al., 2004) quantifies how motivated patients are in taking care of their health. Based on the responses to a set of 13 questions, PAM assigns a level between 1 and 4 . Level 1 indicates that the patient is overwhelmed, while level 4 indicates that the patient is motivated to participate in self-care.

B)Familiarity with the health issue : This feature takes into account different factors that contribute towards a patient's understanding of their health - i) The health literacy of the patient, which represents the ability of a patient to read and understand general health information, as measured by the REALM metric (Davis et al., 1993) ; ii) Patient's prior experience - either because of their own sufferings or because someone in their family had the same issue; iii) Patient's self-assessment of their health knowledge, as obtained from some of the questions in the PAM. 
While the developers of the PAM provide some instructions on how to address patients at different PAM levels ${ }^{1}$, no systematic study of these patients and their views of their illness exist. Under the assumption that such a study can reveal features useful to personalize our summaries, we conducted interviews with 26 patients, who were also asked to answer the PAM questionnaire. In this paper, we describe the quantitative analyses that we have performed on those patient interviews. We start with differences in the terms that those patients use for recounting their health experiences. We found that in spite of using fewer medical terms, patients with high PAM levels speak a higher proportion of unique medical terms. Our analysis of the patients' use of pronouns suggests that patients with low PAM levels tend to selffocus, which is associated with negative effects and low self-confidence (Duval and Wicklund, 1972; Pyszczynski and Greenberg, 1987). Finally, we discuss themes that emerge from those conversations, with the goal of highlighting aspects that hold significance in the patients' lives: for example, patients with high PAM focus more on activities they are interested in, patients with low PAM on their own feelings (confirming the finding about pronouns just discussed).

\section{Related Work}

While several systems exist that summarize medical content (Scott et al., 2013; Pauws et al., 2019), only a few of them produce personalized summaries (Mahamood and Reiter, 2011). Unlike these systems that focus on data-to-text summarization, our personalized summary generation system combines the information from physician and nursing documents and provides hospitalization information to patients in a form that they can understand. Even though a lot of studies have focused on verifying the reliability of the PAM metric (Fowles et al., 2009), no work uses it to produce personalized content for patients. Most of the existing qualitative studies on the narratives of heart failure patients (Jeon et al., 2010; Seah et al., 2016) focus on identifying the factors that impact the patient's self-care and self-management skills. However, none of these studies has looked into the relationship between the content spoken by the pa-

\footnotetext{
${ }^{1}$ https://participatorymedicine.org/epatients/2011/10/the - patient-activation-measure-pam-a-framework-for - developing-patient-engagement.html
}

tients and their motivation to participate in selfcare. Our quantitative analyses are inspired by Pennebaker (2003) and are similar to the studies that predict the empathy of the counselor based on the words used during the session (Althoff et al., 2016; Pérez-Rosas et al., 2017; Xiao et al., 2014).

\section{Interview Collection}

\begin{tabular}{|l|c|}
\hline Category & Values \\
\hline Avg. number of words in an interview(P) & 1655 \\
\hline Avg. number of words in an interview (I) & 1104 \\
\hline Avg. number of words/utterance (P) & 8 \\
\hline Avg. number of words/utterance (I) & 6 \\
\hline Number of low PAM patients & 14 \\
\hline Number of high PAM patients & 12 \\
\hline
\end{tabular}

Table 1: Distributional analysis of the interviews (P: Patient, I: Interviewer)

Since there are no existing publicly available data sets that provide information on the experiences of heart-failure patients, we proceeded to collect one. ${ }^{2}$ We interviewed 26 patients (age range $20-70$ years, $58 \%$ females) who were hospitalized because of heart issues (snippets of an interview can be found in Appendix A). These 50 minutes long open-ended interview sessions were led by a sociolinguist and were later transcribed by professional transcribers. In general, each interview consists of the following stages: 1) Patients are asked to provide their demographic information; 2) Patients are asked to recount their first experience with heart issues, which often leads to them talking about many other issues related to life-style or family; 3) Patients are asked about the recent hospitalization and their experiences; 4) $\mathrm{Pa}$ tients answer the PAM questions; 5) If interested, patients talk about their interests or have a general conversation with the interviewer. For our analyses, we group the patients with PAM level 1 or 2 and refer to them as low PAM patients, while we refer to the group of patients with PAM levels 3 or 4 as high PAM patients. The general statistics on the interviews is shown in Table 1.

\section{Distinguishing Low from High PAM Patients}

We extracted several features from the transcripts, including the counts of different part-of-speech

\footnotetext{
${ }^{2}$ The data is not sharable because of human subject protection constraints, especially as dictated by HIPAA (the Health Insurance Portability and Accountability Act of 1996), United States legislation regarding the safeguard of private health care information.
} 
tags, total positive and negative words, and medical and non-medical type-token ratio (TTR) (i.e. ratio of the number of unique words to the number of words). We then used a Random Forest based approach $^{3}$ for determining the importance of each feature in predicting the PAM level. This process identified some significant features, which are further analyzed in Section 4.1 and Section 4.2.

\subsection{Usage of medical terms}

We extracted the average number of words spoken by the group of low and high PAM patients separately, including the number of medical terms (extracted using cTAKES tool (Savova et al., 2010) ), TTR, and medical TTR. We found that patients with low PAM speak more but have lower value for TTR. Similarly, patients with low PAM use more medical terms, which account for $8 \%$ of their words; for patients with high PAM, medical terms constitute $6 \%$ of their total words, but they were found to have higher medical TTR. Although none of these differences is statistically significant, they still suggest that there is a difference in the lexical diversity (both general and medical) of the two groups of patients.

\subsection{Patient outlook}

\subsubsection{Reference to self}

Researchers on human psychology mention that when individuals start to focus their attention on themselves, they step into a self-evaluative process, where they compare their present to where they aspire to be. For those cases where the present lags behind the aspired standard, selffocus produces a negative effect (Duval and Wicklund, 1972; Pyszczynski and Greenberg, 1987). Similarly, the PAM metric characterizes a patient with low PAM score as an individual who is overwhelmed and weighed down by negative emotions. On the other hand, patients with high PAM are ready to take on challenges and make efforts to improve their health. Hence, in order to verify whether patients with low PAM focus more on themselves, we compared the relative amount of first person singular pronoun vs second and third person pronouns (both singular and plural) used by low and high PAM patients. We split each patient transcript into five parts and observe the trend in the reference to self. As seen in Figure 2, a greater

\footnotetext{
${ }^{3}$ https://scikit-learn.org/stable/modules/generated/sklearn .ensemble.ExtraTreesClassifier.html
}

amount of self-focus is indeed associated with patients with low PAM. The differences across the five parts are statistically significant with a sign test $(\mathrm{z}$-value $=2.23607, p=.02535)$.

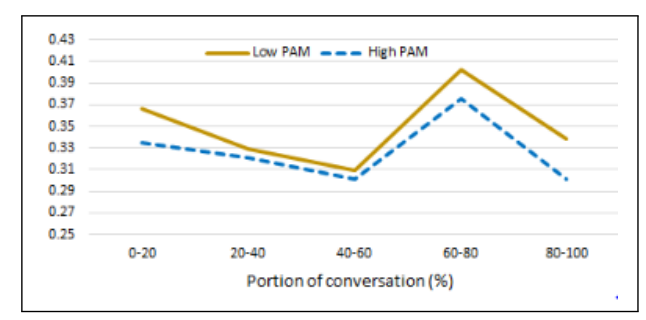

Figure 2: Relative use of first person (singular) vs second and third person pronouns by the patients.

\subsubsection{Sentiment of the patient}

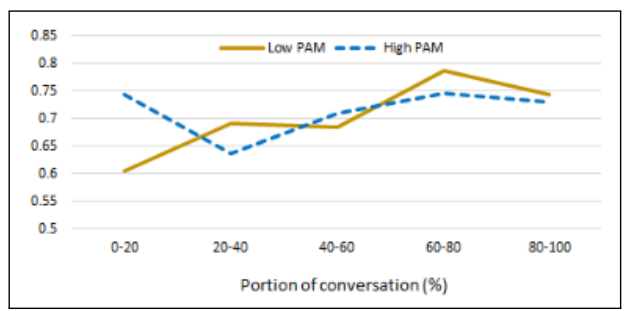

Figure 3: Relative fraction of positive sentences used by patients.

In order to determine how the sentiment of the patients change throughout the conversation, we used the VADER ${ }^{4}$ tool for performing sentiment analysis. Figure 3 shows the relative fraction of positive sentences that are spoken by patients. Interestingly, we can see that the curve for high PAM patients drops during $20-40 \%$ of the conversation, while the plot for low PAM patients drops during $40-60 \%$ of the conversation. One reason behind this is because at around $20-40 \%$ of the conversation, patients are asked to describe their first encounter with heart issues, while at around 40-60\% of the conversation, patients are asked about their current reason behind hospitalization (as was mentioned in Section 3). We can also see that high PAM patients are fairly constant as concerns the fraction of positive content spoken after the first $20-40 \%$ of conversation, while the curve for low PAM patients has more rises and falls. This further supports the observation made by the developers of PAM that low PAM patients are overwhelmed.

\footnotetext{
${ }^{4}$ https://github.com/cjhutto/vaderSentiment
} 


\subsection{Topics Discussed by Patients and Their Relation to PAM Level}

In order to identify the possible themes that arise from the conversations, we extracted only the nouns and adjectives from the patient utterances that occur in at least $10 \%$ of the transcripts and created a document matrix. We then performed Principal Component Analysis (PCA), along with Varimax rotation ${ }^{5}$ on the document matrix. We opted for 9 components (also known as factor loadings) that were able to explain around $75 \%$ of the total variance in the document matrix. Similar to (Wilson et al., 2016), we consider any word with a factor loading of at least 0.2 for a particular component as a positive contributor and words whose factor loadings are less than -0.2 as negative contributors. For each component, we calculate the normalized count of the words from each document that are positive contributors minus the number of negative contributors, and find the averages for low and high PAM patients. Finally, we calculate a score for each component, which is the ratio of average normalized count for high PAM to low PAM patients. A score $>1$ indicates the prevalence of the category in high PAM, while a score $<1$ indicates its prevalence in low PAM patients.

\begin{tabular}{|l|l|l|}
\hline Topic & Sample words & Score \\
\hline $\begin{array}{l}\text { Activities } \\
\text { of interest }\end{array}$ & $\begin{array}{l}\text { ball, game, park,love, physical, } \\
\text { recipe, swimming }\end{array}$ & 2.59 \\
\hline $\begin{array}{l}\text { Technical } \\
\text { medical terms }\end{array}$ & $\begin{array}{l}\text { lasix, murmur, supplement, } \\
\text { admission, sign, diet, specialist }\end{array}$ & 2.55 \\
\hline $\begin{array}{l}\text { Family and } \\
\text { support }\end{array}$ & $\begin{array}{l}\text { husband, family, kind, everybody, } \\
\text { father, parent, support }\end{array}$ & 1.55 \\
\hline $\begin{array}{l}\text { Life } \\
\text { experiences }\end{array}$ & $\begin{array}{l}\text { plan, jump, vacation, swimming, } \\
\text { talk,breath, experience }\end{array}$ & 1.02 \\
\hline $\begin{array}{l}\text { Family and } \\
\text { beliefs }\end{array}$ & $\begin{array}{l}\text { niece,church, grandkid, grandma, } \\
\text { honest, truth, folk }\end{array}$ & 0.95 \\
\hline $\begin{array}{l}\text { Life } \\
\text { experiences }\end{array}$ & $\begin{array}{l}\text { shower, shop,sugar, experience, } \\
\text { contact, downtown, longtime }\end{array}$ & 0.78 \\
\hline Feelings & $\begin{array}{l}\text { terrible, difficult, horrible, } \\
\text { difference, teaching, dizzy,force }\end{array}$ & 0.77 \\
\hline Health & $\begin{array}{l}\text { muscle, workout, lunch, healthy, } \\
\text { vegetable, chicken, information }\end{array}$ & 0.62 \\
\hline Food & $\begin{array}{l}\text { bake, turkey, potato, vegetable, } \\
\text { hot, green, meat, taste }\end{array}$ & 0.21 \\
\hline
\end{tabular}

Table 2: Sample words that reflect the 9 categories/themes, along with the topic scores.

Table 2 shows the sample words that represent the 9 categories/themes and the corresponding scores. The topics in the first column of the table are manual interpretations of what the sam-

\footnotetext{
${ }^{5}$ Varimax rotation causes the weights in the principal components to be closely associated to only one component, which makes it easier to interpret the results of PCA.
}

ple words refer to. The topics with scores greater than 1 are prominent for high PAM patients, while the ones with scores less than one are prominent for low PAM patients. Some interesting observations can be made from this table. First, high PAM patients seem to make more use of technical medical terms, which complements our finding in Section 4.1 that they are less repetitive in their usage of medical terms. Second, patients with low PAM seem to talk about their feelings, most of which relate to the negative effects of their health conditions, while high PAM patients talk about the activities they are interested in. This supports our findings from Section 4.2.1 and Section 4.2.2, which showed that patients with low PAM focus more on the negative changes in their lives.

\section{Conclusion and Future Work}

In this paper, we presented a quantitative study on the interviews with heart failure patients we collected. We analyzed the difference between patients with low and high PAM levels based on their reference to self, usage of medical terms, and the change in their sentiment through the conversation. We also identified the key topics that the patients from both groups talk about. The findings from these analyses have provided additional insights into the characteristics of heart failure patients and will be used for tuning different aspects of our personalized summary generation system.

Incorporating personalization features: Currently, our personalization algorithm provides different levels of details to patients depending upon their familiarity with their health issues. Similarly, for patients with a low PAM level, we show empathy with sentences like "Dealing with this issue must have been tough for you", while high PAM patients are provided encouragement with sentences like "Keep up the good work".

From the analyses of the usage of medical terms (Section 4.1) and the topics discussed by patients (Section 4.3), we found that the PAM level of a patient is also an indicator of the type and amount of medical terms that patients use while recounting their health experience. This suggests that in addition to health literacy (as we do currently), the PAM level should also be taken into account for deciding on the details that will be provided to the patient. Based on the findings in Section 4.2.1 and Section 4.2.2, we plan to divert low PAM patients from self-focus and its potential negative ef- 
fects, for example by focusing more on positive outcomes and improvements in their health status. We also plan to use some of the topics that were discovered in Section 4.3 as multiple choice questions that will be shown to patients in real time. Based on the values that are selected, some generic sentences that motivate the patients to get better will be included in the summary.

\section{Acknowledgments}

This work was supported by award R01 CA225446-01 from the National Cancer Institute.

\section{References}

Sabita Acharya, Andrew Boyd, Richard Cameron, Karen Dunn Lopez, Pamela Martyn-Nemeth, Carolyn Dickens, Amer Ardati, and Barbara Di Eugenio. 2019. Incorporating personalization features in a hospital-stay summary generation system. In Proceedings of the 52nd Hawaii International Conference on System Sciences.

Sabita Acharya, Andrew D Boyd, Richard Cameron, Karen Dunn Lopez, Pamela Martyn-Nemeth, Carolyn Dickens, Amer Ardati, Jose D Flores, Matt Baumann, Betty Welland, et al. 2018. What happened to me while i was in the hospital? challenges and opportunities for generating patient-friendly hospitalization summaries. Journal of Healthcare Informatics Research, pages 1-17.

Sabita Acharya, Barbara Di Eugenio, Andrew D Boyd, Karen Dunn Lopez, Richard Cameron, and Gail M Keenan. 2016. Generating summaries of hospitalizations: A new metric to assess the complexity of medical terms and their definitions. In The 9th International Natural Language Generation conference, page 26.

Tim Althoff, Kevin Clark, and Jure Leskovec. 2016. Large-scale analysis of counseling conversations: An application of Natural Language Processing to mental health. Transactions of the Association for Computational Linguistics, 4:463-476.

Terry C Davis, Sandra W Long, Robert H Jackson, EJ Mayeaux, Ronald B George, Peggy W Murphy, and Michael A Crouch. 1993. Rapid estimate of adult literacy in medicine: a shortened screening instrument. Family medicine, 25(6):391-395.

Barbara Di Eugenio, Andrew D Boyd, Camillo Lugaresi, Abhinaya Balasubramanian, Gail M Keenan, Mike Burton, Tamara G Rezende Macieira, Karen Dunn Lopez, Carol Friedman, Jianrong Li, et al. 2014. Patientnarr: Towards generating patientcentric summaries of hospital stays. INLG 2014, page 6 .
Shelley Duval and Robert A. Wicklund. 1972. A theory of objective self awareness.

Jinnet Briggs Fowles, Paul Terry, Min Xi, Judith Hibbard, Christine Taddy Bloom, and Lisa Harvey. 2009. Measuring self-management of patients and employees health: further validation of the patient activation measure (pam) based on its relation to employee characteristics. Patient education and counseling, 77(1):116-122.

Martha M. Funnell. 2000. Helping patients take charge of their chronic illnesses. Family practice management, 7(3):47.

Judith H Hibbard, Jean Stockard, Eldon R Mahoney, and Martin Tusler. 2004. Development of the patient activation measure (pam): conceptualizing and measuring activation in patients and consumers. Health services research, 39(4p1):1005-1026.

Yun-Hee Jeon, Stefan G Kraus, Tanisha Jowsey, and Nicholas J Glasgow. 2010. The experience of living with chronic heart failure: a narrative review of qualitative studies. BMC health services research, 10(1):77.

Saad Mahamood and Ehud Reiter. 2011. Generating affective natural language for parents of neonatal infants. In Proceedings of the 13th European Workshop on Natural Language Generation, pages 1221. Association for Computational Linguistics.

J Michael McGinnis, Leigh Stuckhardt, Robert Saunders, Mark Smith, et al. 2013. Best care at lower cost: the path to continuously learning health care in America. National Academies Press.

Steffen Pauws, Albert Gatt, Emiel Krahmer, and Ehud Reiter. 2019. Making effective use of healthcare data using data-to-text technology. In Data Science for Healthcare, pages 119-145. Springer.

James W Pennebaker, Matthias R Mehl, and Kate G Niederhoffer. 2003. Psychological aspects of natural language use: Our words, our selves. Annual review of psychology, 54(1):547-577.

Verónica Pérez-Rosas, Rada Mihalcea, Kenneth Resnicow, Satinder Singh, and Lawrence An. 2017. Understanding and predicting empathic behavior in counseling therapy. In Proceedings of the 55th Annual Meeting of the Association for Computational Linguistics (Volume 1: Long Papers), volume 1, pages 1426-1435.

Tom Pyszczynski and Jeff Greenberg. 1987. Selfregulatory perseveration and the depressive selffocusing style: a self-awareness theory of reactive depression. Psychological bulletin, 102(1):122.

Barbara Riegel, Christopher S Lee, Nancy Albert, Terry Lennie, Misook Chung, Eun Kyeung Song, Brooke Bentley, Seongkum Heo, Linda WorrallCarter, and Debra K Moser. 2011. From novice to expert: confidence and activity status determine 
heart failure self-care performance. Nursing research, 60(2):132-138.

Guergana K Savova, James J Masanz, Philip V Ogren, Jiaping Zheng, Sunghwan Sohn, Karin C KipperSchuler, and Christopher G Chute. 2010. Mayo clinical text analysis and knowledge extraction system (ctakes): architecture, component evaluation and applications. Journal of the American Medical Informatics Association, 17(5):507-513.

Donia Scott, Catalina Hallett, and Rachel Fettiplace. 2013. Data-to-text summarisation of patient records: Using computer-generated summaries to access patient histories. Patient education and counseling, 92(2):153-159.

Alvin Chuen Wei Seah, Khoon Kiat Tan, Juvena Chew Huang Gan, and Wenru Wang. 2016. Experiences of patients living with heart failure: a descriptive qualitative study. Journal of Transcultural Nursing, 27(4):392-399.

Johanna Shapiro. 1993. The use of narrative in the doctor-patient encounter. Family Systems Medicine, 11(1):47.

Steven R Wilson, Rada Mihalcea, Ryan L Boyd, and James W Pennebaker. 2016. Cultural influences on the measurement of personal values through words. In 2016 AAAI Spring Symposium Series.

Bo Xiao, Daniel Bone, Maarten Van Segbroeck, Zac E Imel, David C Atkins, Panayiotis G Georgiou, and Shrikanth S Narayanan. 2014. Modeling therapist empathy through prosody in drug addiction counseling. In Fifteenth Annual Conference of the International Speech Communication Association. 


\section{A Supplemental Material}

Patient: Quality of life is more valuable to me than working on the next project

Interviewer: Alright. Okay. So self care...

Patient: matters

Interviewer: Is primary. Yeah it matters. Yeah definitely.

Patient: Yeah

Interviewer: Now do you have some support at home? You girlfriend's around? She is going to help you out or?

Patient: Yeah. You know I am probably the most fortunate guy you are gonna meet today. I am fortunate in that even aside from my family, there are friends that genuinely care about me.

Interviewer: Alright

Patient: I mean really genuinely..genuinely care about me

Interviewer: Ah

Patient: Care about what I do..care about my well being..care about who I am...what I do..I have friends that will not allow me to fail.

Figure 4: A portion of an interview where the patient talks about the things that matter to him .

Patient: Ahm hm. Yeah. So they give you this Lasix they try to get that fluid and stuff off your lungs that makes your legs swell too.

Interviewer: Ah hm. And is that water part of the heart thing? The heart problem or?

Patient: Right. Ah hm

Interviewer: What is this Lasix thing? What is that?

Patient: it is a shot they give you. In fact they give you pills too... So they will bring water..you know..out of your body

Figure 5: A portion of an interview where the patient explains about her health issue.

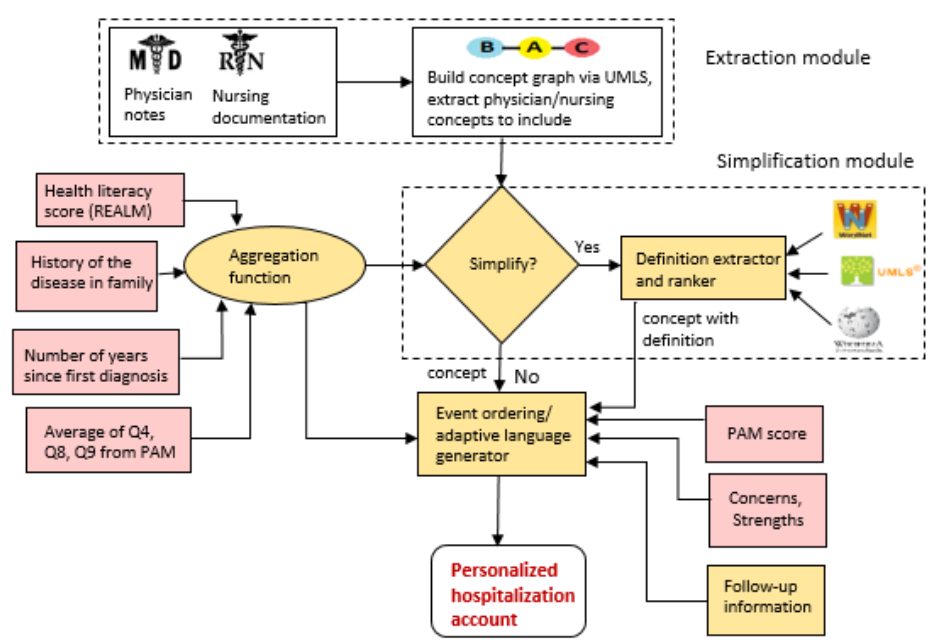

Figure 6: Work-flow of the algorithm for generating personalized summaries. Extraction module is responsible for exploring the relationship between the medical terms from the input documents. Simplification module identifies difficult medical terms and provides explanations to them. The boxes in pink represent the features that guide the personalization process. 